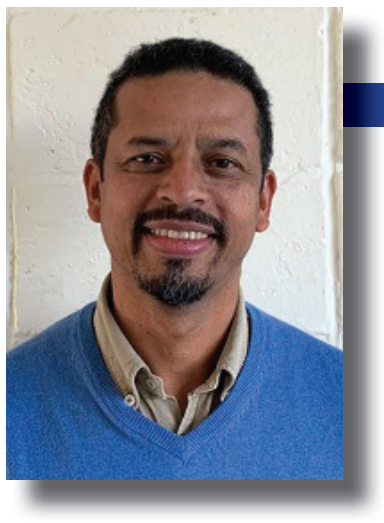

\title{
HACIA UNA UNIVERSIDAD \\ DE ALTERNATIVAS
}

\author{
PhD. ROQUE CASTRO SUAREZ \\ rocasuarez@gmail.com \\ Departamento de Socología, UNAH \\ Tegucigalpa, Honduras \\ ORCID: 0000-0002-2284-8040
}

DOI: $10.5377 /$ rpdd.v4i1.9384

Recibido: febrero, 2017

Aceptado: agosto, 2017

\section{RESUMEN}

En este artículo se exponen algunos de los aspectos fundamentales de la relación universidad sociedad en el contexto de la reforma universitaria de la Universidad Nacional Autónoma de Honduras. Desde la perspectiva democrática emancipadora se analizan las relaciones universidad sociedad entre el año 2004 y 2015. Se critican los límites de la reforma y se proponen alternativas desde la proyección social de la universidad y desde la formación de sujetos

\section{ASBTRACT}

This article discusses some of the fundamental aspects of the relationship university society in the context of reform. From a democratic perspective emancipatory university relations in society between 2004 and 2015 analyzes the limits of reform is criticize and propose alternatives from the social of the university and from the formation of subjects.

\section{INTRODUCCIÓN.}

Este artículo se sintetizan los principales aspectos de la tesis de doctorado: universidad sociedad en el contexto de la reforma. El caso de la, Universidad Nacional Autónoma de Honduras-UNAH (2004-2015). Es un estudio de características cualitativas realizado sobre la base de entrevistas. El tema reviste importancia en tanto no existen estudios sobre la UNAH que aborden críticamente el camino recorrido por la universidad en la últimas décadas (sobre todo en el periodo de la reforma) y lo que la universidad 
debería ser desde una perspectiva democrático emancipadora que contribuya a la formación de ciudadanos y sujetos frente a las exigencias estructurales de nuestra sociedad. Es de des-tacar que la mayoría de la información y de los argumentos aquí utilizados son anteriores al conflicto universitario (mayo julio de 2016 e incluso a los acontecimientos de julio 2015).

\section{UNIVERSIDAD Y DESARROLLO EN AMERICA LATINA.}

Estamos en un momento donde el Estado no demanda contribuciones estratégicas (para la democracia y la justicia que requiere el país) ni la sociedad en general se ha acostumbrado a ellas pero también la universidad tampoco las ofrece. La transición no concertada que refleja una reforma limitada solo ha podido ser cuestionada con la crisis universitaria que provoca la protesta estudiantil en el 2016.

Hay que señalar que si bien la universidad ha hecho aportes importantes durante su historia, como ocurre durante el periodo de lo que se considera la universidad tradicional y particularmente en la etapa independentista, la universidad realizo contribuciones importantes a la sociedad, preparando lo funcionarios para los nuevos gobiernos y como uno de los autores señala, para la sociedad de la época, su estructura productiva se satisfacía con la formación de profesionales en las carreras tradicionales (Molina, 2008;132).

Posteriormente, con la formación de los Estados Nacionales (cuando las universidades se reabren después de que durante la independencia varias fueron cerradas) como una universidad subordinada al Estado con funciones, como plantea Tunermann (1991) de adiestramiento cultural y profesional de la elite promoviendo a la vez la unidad y la estabilidad política del Estado.

En una perspectiva similar Marcela Mollis sostiene que en el siglo XIX en el marco de la conformación de los Estados Nacionales la educación juega un papel fundamental en la configuración de la identidad de la nación. A las universidades les corresponde además preparar el personal burocrático que el Estado requiere (Mollis, 2014; 4).

La transición que significa Cordoba hacia la universidad modernizante, como universidades masivas sobre todo en su etapa madura, con nuevas exigencias del capital y para el Estado, se presenta por tanto una realidad más compleja que plantea nuevas demandas para la universidad. En este contexto las funciones anteriores se desacoplan, se vuelven insuficientes y es cuando apararen las preocupaciones por el desarrollo donde la educación debe contribuir a mejorar los niveles de productividad aportando además a la movilidad social no solo como presión sociopolítica sino también como requerimiento del modelo de sustitución de importaciones. 
Ahora bien, la reforma de Córdoba es importante, porque contrario a lo que pensaban algunas autoridades de la UNAH, Córdoba, como la ha mostrado el movimiento estudiantil en las luchas entre 2014 y 2015, no es un asunto del pasado. El legado y la memoria de Córdoba es por tanto recreada en estas luchas.

En síntesis Brunner sostiene como rasgos característicos de las universidades modernas latinoamericanas la masificación que tiene lugar entre 1950 y 1980, la diferenciación interna de la universidad, la politización y el conflicto de valores que se dan en la universidad. Admite que las relaciones con la sociedad son cambiantes y complejas. Quizá por otro lado es posible señalar que la universidad latinoamericana de este periodo ha sido un importante espacio de integración social, aunque en general su vínculo con el desarrollo es débil y más cuando se trata de contribuciones importantes para superar las desigualdades y la pobreza de la región latinoamericana.

Un tema importante a considerar es que la idea de desarrollo aquí es presentada en su carácter contra hegemónico y en su versión más radical, según la perspectiva se Sousa Santos, se plantea como la búsqueda de alternativas ya no de desarrollo sino como alternativas al desarrollo. El desafío es por tanto abrirse a otras posibilidades recuperando las experiencias desperdiciadas o silenciadas desde el dialogo de saberes. Es necesario experimentar en busca de alternativas.

\section{UNIVERSIDAD Y SOCIEDAD EN HONDURAS}

Mario Posas (2014) distingue las siguientes etapas como acercamiento a la historia de la universidad nacional: la primera de ellas comprende desde la fundación en 1847 hasta el Reforma Liberal de 1876 que se considera como un periodo de marcada influencia de la Iglesia Católica y en buena medida por el Estado. Una segunda etapa, desde la reforma liberal en 1876 hasta 1948 cuando la universidad adquiere características más laicas y se ofrecen carreras consideradas liberales. Una tercera etapa comprende desde 1949 como un periodo de modernización económica e institucional hasta 2004, donde la universidad adquiere la autonomía y se aprueba la paridad estudiantil. Es además un periodo de expansión de la oferta de nuevas carreras y de demanda estudiantil.

Esta última etapa es subdividida por el autor en un periodo que va de 1949, donde el Estado es protagonista de una modernización y de desarrollo sin que se excluyan momentos de inestabilidad política que se manifiestan en golpes de Estado, hasta 1982. Se considera un periodo de gran politización donde sectores de centro izquierda gobiernan la universidad. Un segundo momento va de 1982 hasta 2004 donde sectores conservadores gobiernan la universidad nacional. Hay que destacar sin embargo que a partir de 2004 se inicia el periodo de transición como la etapa que 
aquí interesa analizar.

\section{PROYECTOS DE UNIVERSIDAD EN DISPUTA.}

En esta etapa (1949-2004) dos proyectos de universidad se disputan la hegemonía, es decir, el control de la universidad. Uno es el proyecto conservador donde hay un apoyo directo del Estado en el marco de la Doctrina para la Seguridad Nacional y en relación al proyecto progresista el apoyo del Estado es más indirecto afectado por las fuerzas de izquierda que están movilizadas en el continente. Estos dos proyectos como antecedentes, inciden en el proceso de reforma en tanto tensionan la disputa del mismo. Mientras la versión hegemónica de la reforma (2004-2016), como proyecto de universidad actual, combate y busca superar los dos proyectos en disputa, con algún apoyo del Estado y de sectores políticos en el poder (que entre otros aspectos lo hacen converger con modelo conservador), los agentes que presionan y critican el modelo de reforma dominante (proyecto emergente), activan su disputa desde la memoria del modelo progresista y fundamentalmente desde los nuevos códigos de indignación juvenil del presente.

Según uno de los entrevistados en el proyecto progresista se destaca el apoyo del Estado en estos procesos y como también la migración de académicos del sur contribuyeron a posicionar el pensamiento crítico, que con profesores de clase media en conjunto apostaron por la reforma que, inspirada, aunque de forma tardía en la tradición de Córdoba. En este periodo la universidad busca abrir vínculos con la sociedad y principalmente a los sectores sociales aunque no se trata de una vinculación estratégica sistemática y que como tal posteriormente fue desmantelada por fuerzas conservadoras.

Uno de los aspectos que llama la atención en este periodo es que se realizaron dos encuentros universitarios. Uno celebrado del 12 al 16 de noviembre de 1974 cuyo lema era el papel de la universidad cosiste en contribuir a la transformación social y a la liberación nacional. Es un lema consecuente con el periodo donde las fuerzas progresistas apostaban por un cambio revolucionario. El segundo encuentro se realizó del 11 al 15 de mayo de 1981 y cuyos objetivos se centraron en analizar las relaciones con el gobierno central y con los diversos sectores de la sociedad.

Se puede observar como en ambos periodos la agenda dominante estaba centrada en factores externos, como característica central del contexto, sean estos vinculados con el Estado y o con la sociedad. La proyección social se vuelve central, la universidad está volcada hacia la sociedad.

Un tercer encuentro tiene lugar del 4 al 8 de septiembre de 1989 y la preocupación central fue la "excelencia académica". Fueron los momentos cuando la 
cuestión de la calidad de la educación aparecía en el debate, inicialmente propuesto por sectores de izquierda y posteriormente abordado por los organismos internacionales, cuando la guerra fría finalizaba y el neoliberalismo sentaba sus bases. Estamos obviamente en un nuevo gobierno de la universidad.

Es oportuno señalar que el tema de concertación y de los pactos universitarios como tesis central de este artículo, en tanto contribuye a generar un cambio sostenible y profundo orientado a una sociedad más incluyente y más democrática, es como se puede ver, convergente con los encuentros universitarios que forman parte de la tradición universitaria. Implica además que no se rompe con la historia, contrario al estilo de la actual gestión, sino que se recupera lo mejor que hay en ella.

Una docente también plantea como en la rectoría de Juan Almendares, como gobierno progresista, ya se ha instalado la crisis y donde factores internos como el papel que juega el sindicato serán de importancia. Es el momento cuando el Frente Unido Universitario apoyado por todas las fuerzas conservadoras del país se coloca al frente de la Federación de Estudiantes Universitarios de Honduras (FEUH).

La FEUH después de ser una instancia de representación estudiantil con mayor prestigio y con niveles de responsabilidad social, paso a ser en el gobierno conservador, un espacio de corrupción y con escasa legitimidad pues desde entonces nunca tuvieron lugar elecciones estudiantiles reconocidas y aceptadas por los estudiantes y por toda la comunidad universitaria. Las "elecciones" estudiantiles en los años 80 principalmente, estuvieron siempre marcadas por su control armado por parte de los sectores más conservadores de la universidad quienes a su vez estaban protegidos por el Estado hondureño en el marco de la Doctrina de la Seguridad Nacional.

Hay que señalar que el control que se tenía o se ejercía desde la FEUH será fundamental para mantener el poder en la universidad porque la FEUH, en tiempos de la paridad estudiantil, representaba el $50 \%$ de los votos en el Claustro Pleno en general controlado por los sectores más conservadores de la universidad. En este contexto la FEUH pierde legitimidad y su prestigio lo que consecuentemente se convierte en el argumento para los reformadores de 2004 de proponer el cambio y anular la paridad estudiantil que se contemplara luego en la nueva Ley Orgánica de la universidad.

Si bien varios docentes y académicos destacan también las contribuciones y el ambiente de este periodo de universidad progresista y por otro lado, la gestión conservadora que se instala en la universidad, se destaca, en el periodo progresista, el protagonismo de los estudiantes donde varios de ellos como consecuencia de su 
compromiso, van a vivir persecución y la desaparición forzada. Al mismo tiempo se menciona como en medio de la crisis surge un sector de docentes que será el promotor del proceso de transición que se inicia en el año 2004.

Mientras se añora el pasado en cuanto este represento espacios importantes para el debate y el análisis de temas considerados relevantes y con la participación de actores también relevantes, donde la universidad se pronuncia y esta se consideraba más cercana a la realidad de las mayorías populares y pese a todo esto, un entrevistado reconoce que hay sectores, durante los gobiernos progresistas, que son marginados. Esto supone que la lógica del poder, en uno y otro proyecto (hasta el actual), es excluyente y eso hace difícil los pactos y acuerdos. Por tanto la cultura del dialogo y de las decisiones democráticas, si se valora sus ventajas para el cambio sostenible, es algo que hay que construir. Este proceso fue el que se abrió después de la crisis de 2016.

Como se puede observar por otro lado, según la perspectiva de algunos docentes, se plantean ciertos aspectos que es importante considerar en tanto aporta otros elementos que permiten ver la complejidad y profundidad de estos procesos. Uno de esos aspectos es como alrededor del movimiento estudiantil y la apertura al pensamiento crítico se permitía un mayor debate en la universidad. La universidad se pronunciaba sobre temas nacionales, era una universidad más cercana a la problemática nacional y la sociedad escuchaba lo que decía la universidad. Según uno de los entrevistados, esta universidad, con sus errores y virtudes, es preferible a la actual. De alguna forma se plantea que no se puede romper abruptamente con este pasado de la universidad tal como pretenden las actuales autoridades.

Otro de los aspectos que aporta un entrevistado y que puede considerarse de importancia es como ciertos docentes de la ADUNAH apoyaron el proceso de transición que se inicia en el año 2004 pero que no convergen cuando se va formando un gobierno que los sectores más críticos consideran autoritario (, surgen grupos de control, se destruye el sindicato y se golpea a la ADUNAH) y las nuevas estructuras de poder cuando surge la Comisión de Transición. El docente critica la ilegalidad que se genera en el Consejo Universitario cuando lo delegados se nombran de dedo, no hay representación estudiantil y luego este consejo crea e impone un reglamento electoral estudiantil. En este contexto es cuando se inicia una lucha por el poder y los que no están con las autoridades son considerados anti reformistas.

Hay que considerar que el movimiento estudiantil progresista llega muy debilitado a los años 90 y 2000 por todos los impactos de la represión y la desaparición de algunos de sus miembros y tiene que enfrentar no solo los efectos de una nueva coyuntura de asesinatos selectivos sino también los efectos más globales 
del neoliberalismo. Favorablemente el Golpe de Estado de 2009 logro articular al movimiento estudiantil (lo poco que quedada) y politizo muchos estudiantes que conformaron y convergieron en movimientos independientes. Estos últimos han sido los protagonistas con fuerte apoyo de la población estudiantil, particularmente en los conflictos más recientes de los años 2015 y 2016.

Uno de los temas críticos que ha hecho difícil la representación estudiantil es la división entre frentes estudiantiles, los movimientos independientes y las asociaciones de estudiantes (las convergencias han sido pocas). Esta división ha dificultado la realización de elecciones después de varios intentos pero también las alianzas de los frentes tradicionales considerados de izquierda (FRU y FUR) con las autoridades. Las autoridades le han sacado provecho a estas limitaciones, las han alimentado cuando ha sido posible y han buscado fórmulas de representación que les son favorables.

Por otro lado, en relación a los proyectos antecedentes, se destaca además el impacto negativo que la lucha político ideológico género para el desarrollo de un proyecto académico compartido por la comunidad universitaria. La proyección social no fue sistemática y la articulación de las funciones tradicionales de la universidad fue también débil.

Hay que destacar que el tema político que es una constante de la historia de las universidades latinoamericanas aparece aquí en la disputa de los modelos de gestión de la universidad. Lo anterior se relaciona con la necesidad de acuerdos y pactos como una forma de colocar en debate racional la disputa de proyectos, como un asunto propio de la razón de ser de la universidad y como forma de pedagogizar la gestión universitaria. Al mismo tiempo la universidad debe discutir sobre la necesidad de la mejor política tanto hacia adentro como hacia afuera, sobre lo que significa hacer política de forma universitaria. Todo ello por tanto está vinculado al tema de la ciudadanía que las universidades están obligadas a fortalecer como forma consciente porque de todas formas la universidad inconscientemente incide en la formación de ciudadanías restringidas cuando no tiene un proyecto estratégico al respecto.

También, además de constatar el impacto político ideológico del periodo progresista, y la politización partidaria de la gestión conservadora, otro docente y miembro del Consejo Universitario plantea como la autonomía universitaria fue burlada por los partidos en el poder hasta llegar a la corrupción y el deterioro académico. La universidad perdió legitimidad.

Se observa que el entrevistado también hace una crítica tanto al gobierno universitario progresista como lo hace también con el modelo conservador. Sin 
embargo, hay que indicar que en la medida que realiza esa crítica destaca la actual gestión y consecuentemente deja de reconocer varios aspectos de la tradición universitaria. Entre los aspectos positivos es importante destacar que iniciar un proceso de transición con un fortalecimiento institucional aunque sea desde arriba es un avance. El reto pasa por consolidar ese proceso desde la idea de un proyecto académico compartido.

La politización, la fuerte presencia partidaria como la ideologización serán rasgos dominantes de estos periodos, constituyéndose en elementos importantes de la tradición universitaria. Hay que destacar que mientras en los dos primeros modelos encontramos una forma de política polarizada de dos proyectos en contienda propios del periodo, en la actual etapa esa tendencia, por el debilitamiento y la crisis de los actores tradicionales de la universidad, por su débil resistencia, más la difícil emergencia de otros con capacidad de generar oposición (hasta la crisis de 2016), se impone un modelo unilateral que tiende a la anulación de la política o la despolitización.

Esta tendencia de anulación de la política y/o la despolitización en tanto no se establecen condiciones para una politización positiva propia de una formación de sujetos y/o de ciudadanos acaba generándose una politización negativa que lleva a una forma de ciudadanías de baja intensidad y/o restringidas, ${ }^{1}$ aunque paradójicamente también se abren espacios de democratización como ocurre actualmente en la UNAH.

\section{Los límites de la reforma en la UNAH.}

La reforma que se inicia en 2004 (autoevaluación de carreras, planes de mejoras, evaluación de pares y acreditación) tiene límites en cuanto su llamado momento conceptual y la socialización del modelo educativo no fue tal. Una de las responsabilidades según las autoridades recae sobre las unidades académicas pero desde arriba no se idearon los equipos para tal fin. Además por la influencia externa del modelo que sigue la lógica de la globalización competitiva, la autonomía se fe afectada cuando no hay debate ni procesos participativos. No obstante el movimiento del cambio es fundamentalmente interno.

\footnotetext{
El esquema es tomado de lo que Isaías Berlín llama la idea de libertad negativa y libertad positiva pero orientada en otra dirección. Politización positiva implica la apertura institucional y la creación de condiciones para la buena política, para la participación democrática. La politización negativa es aquella que es inhibida y restringida por las instituciones y genera como efecto conflictos que paradójicamente abren o pueden abrir espacios democráticos. La experiencia parece indicar que cuando la politización positiva es imposible la politización negativa es necesaria, sobre todo, en el sentido de abrir desde abajo espacios de participación democrática.
} 
Si bien la reforma ha tenido un avance curricular y supone también otras transformaciones institucionales que aparecen con la aprobación de la nueva Ley Orgánica y las nuevas Normas Académicas, las protestas estudiantiles que vienen desde 2014 hasta 2016 cuestionaran su carácter no concertado y la ausencia de democracia que conlleva el gobierno universitario.

En relación a lo primero presupuesto ha sido uno de los puntos críticos de la autoevaluación y la reforma curricular. El presupuesto es un problema para llevar adelante la reforma. Si en general se desconoce el manejo del presupuesto, hay una especie de control político, manipulación, se desconocen los criterios, no hay transparencia, hay que democratizar el presupuesto (discusión en reunión de planificación de la Facultad de CCSS, 23 abril, 2014), a la reforma curricular no se le asigno presupuesto. No se dio además el tiempo necesario ni se ubicaron equipos que siguieran con proyecto compartido de universidad y sociedad.

Con lo señalado queda claro que en el caso de la Universidad Nacional el currículo es uno de los ejes del cambio siguiendo lo planteado por algunos autores ( $\mathrm{S}$ Bustamante y Sánchez, 2007; 212), y también Tedesco,1992). Sin embargo, hay que reconocer que las opciones de la reforma y de los procesos de cambio son más amplios y en este caso implico reformas institucionales como el Consejo Universitario y las nuevas normas académicas. Pero como lo plantea Peter Mc Laren y Luis Huerta el horizonte del cambio debe ser la humanización emancipación ( Mc Laren y Huerta, 2010; 1124-1129). Además el tema de la formación de ciudadanía y de sujetos es central, su contribución a la justicia social y la democratización es fundamental. Este horizonte no se observa en la reforma universitaria que aunque se haga más investigación y vinculación (todavía no de forma estratégica), sigue atrapada en lo profesionalizante.

Existe y ha existido por tanto un discurso de la reforma como discurso del poder. Por mucho tiempo ha funcionado así (el momento conceptual que plantea la vice rectora académica). Existe todavía más como discurso desde arriba. Revela de esa forma la "ausencia" de un proyecto académico relevante y sostenible. La autoevaluación se ubica dentro de la "función social asignada" a la universidad. Se apela a la reforma como recurso ideológico político, es una reforma inercial, la reforma como promesa, como declaración y como proyecto ha existido por mucho tiempo en la universidad. Como ocurre con sus funciones declarativas, la reforma se convierte también en un recurso de legitimación.

La rectora es consiente que la reforma "intangible" es más difícil que la reforma basada en la infraestructura, en tanto la primera "depende de las voluntades de las carreras, de los profesores". Es por tanto una prueba de fuego para su liderazgo. 
Por otro lado valora que los estudiantes viven la reforma al incrementar el "orgullo universitario, mayor autoestima, están más conscientes de sus derechos". Admite sin embargo que la defensa de la reforma por parte de los estudiantes "es otro cuento" y que probablemente algunos estudiantes si lo hacen. Quizás se sienten las bases para una reforma de mayor aliento si se cumplen las otras condiciones de pactos y acuerdos.

La titular de la rectoría defiende que la consolidación de la reforma es un proceso de mediano plazo, de 10 a 15 años. La crisis profunda que vive el país afecta este proceso. Por otro lado también existe la amenaza de los sectores que gobernaron en el pasado que apuestan por la contrarreforma. Sostiene además que las propuestas de la reforma tienen que salir de cada facultad y que si eso no ocurre, hay que impulsarlas desde afuera.

Según la rectora de la universidad el problema político partidario e ideológico es un obstáculo para avanzar en los procesos de reforma. Si se admite que la historia universitaria reciente está marcada por un sobredimensionamiento ideológico, una ideologización que no pudo universitariamente equilibrar un proyecto académico relevante, tiene razón. Sin embargo, la tradición crítica y comprometida de esa historia que converge con la función originaria, que tienen que ver con la vocación critica, con la negación del poder y como contrapeso del poder, de la universidad, no se puede desconocer. En otras palabras se puede decir que lo ideológico juega no solo en una dirección sino en varias direcciones y por tanto incluye a las autoridades y otros actores externos como el Estado. Reconocer este obstáculo por otro lado implica también ofrecer salidas que legitimen el proceso de cambio y a mediano plazo lo hagan sostenible. El proceso seguido hasta la fecha no parece confirmar esto último.

Muy relacionado con lo anterior es lo que también plantea la rectora cuando sostiene que las universidades públicas se mueven en una lógica de voluntad por sobre las funciones que hay que asumir por derecho. Señala además que los Consejos Locales funcionan con un cierto compadrazgo y que por tanto hay que intervenir en las contrataciones. En relación a lo primero sin embargo es posible caer en la otra voluntad que es la voluntad del poder y no la voluntad de poder en el sentido plantado por Niesche. $Y$ en relación a lo segundo el peligro es que se caiga en otro compadrazgo desde arriba. En consecuencia el poder crea orden y el orden tiene consecuencias.

Uno de los expertos en temas de educación superior también ha sostenido que las principales dificultades no son técnicas, sino ideológicas. En principio todos coinciden en educación democrática, pertinente, de calidad, de responsabilidad pero 
luego aparecen las disputas (Sobrinho, 2008;134). Coincidir sin embargo en que los temas ideológicos son un gran problema para el cambio es importante pero lo decisivo es como se responde a esa situación. El pacto universitario es una posibilidad para el cambio sostenible.

De ahí que existe el peligro de que el ideal de la reforma modernizadora tienda a convertirse en una ideología sobre todo en la alta dirección, que les impide ver con objetividad las dificultades que entraña un verdadero proceso de transición cuando se supone la participación y acompañamiento de la comunidad académica (Quintero: 2013; 336)

Ahora bien, el tema por un lado hace aparecer el conflicto y por el otro si se considera que son "los otros" los que tienen este problema y se ubica fuera de la cuestión ideológica, se está sin duda frente a otro gran problema que hace recordar el viejo asunto que viene del siglo XIX donde los sectores dominantes establecieron que lo ideológico respondía a motivos humanos menos nobles que los de la ciencia (Serrano, 2010; 241) o de los que aducen tener buenas intenciones. Según Dussel toda teoría y práctica en función del encubrimiento de la dominación son ideológicas (Dussel, 2011; 249).

Si bien el problema ideológico impide en las autoridades generar el cambio desde una lógica de un pacto universitario, el tema de la reforma es complejo, pero la misma complejidad justifica la mayor participación de los actores.

Si lo estudiantes son el alma de la universidad, al menos por ser mayorías, lo que se observa en la reforma es su baja participación. Su participación en la reforma curricular ha sido nula. No se diseñó ni en el plan de la vice rectoría ni en las unidades académicas su mejor inclusión. La reforma curricular ha sido un asunto de las comisiones de docentes responsabilizadas para tal fin. Además si se considera que las transformaciones actuales de la universidad se han realizado desde el control del Consejo Universitario con déficit de representación, es un tema central para inclusión estudiantil.

Como es sabido solo después de las protestas estudiantiles de 2015 los estudiantes han sido incluidos en diálogos "académicos", sin incorporar los asuntos que las autoridades consideran "políticos"( reforma democrática, la representación estudiantil, normas académicas, la dirección de la reforma). Hasta la fecha sin embargo no se conocen resultados sobre estos diálogos y donde los estudiantes más críticos tienen pocas expectativas en tanto los temas políticos no están en agenda. Posteriormente (2016) hay que señalar que los conflictos fueron resultados del fracaso del dialogo anterior y por problemas acumulados y los nuevos efectos de las normas 
académicas. Esta vez, después de un prolongado conflicto, se ha firmado un acuerdo de dialogo y se ha instalado una comisión de seguimiento.

En alguna medida hay que decir que la no inclusión en la reforma y los procesos de cambio de la universidad ha llevado a los conflictos y protestas estudiantiles. Lo anterior parece indicar que la reforma desde arriba y excluyente no es sostenible y no tendrá la profundidad que requieren los cambios para una sociedad alternativa. En definitiva la participación de los estudiantes como de los otros actores (como lo docentes no incluidos en estos diálogos) es decisiva si se quiere apostar por cambios relevantes.

Todo lo anterior también plantea la necesidad de la adecuada politización de los universitarios que debe considerar esa larga tradición universitaria que no se puede eludir y por tanto se deben valorar las diferencias de esa politización. El tema es relevante porque se pasó de modelos politizados a otro de características aparentemente "despolitizadas" como es el actual donde se penaliza la protesta y se colocan obstáculos físicos para que la misma no se realice. Asumir esta tarea es importante para lograr el tipo de cambio que aquí se propone y es consecuente con la idea de formación de ciudadanos y la constitución de sujetos como parte de la universidad que se quiere para una sociedad alternativa a la existente de exclusión, de desigualdad, de crisis de convivencia y también crisis de sentido.

Por otro lado, la reforma por carreras aisladas perece un desperdicio. Si bien se ha logrado que unas 16 carreras seleccionadas (como plan piloto de la vice rectoría académica) concluyan su proceso de desarrollo curricular, son procesos aislados donde se atiende la particularidad de las carreras por sobre una perspectiva estratégica que articule creativamente, más allá del modelo profesionalizante que sigue siendo dominante, la proyección social, la investigación y la docencia.

Se cuestiona por otro lado que la evaluación es limitada, en la medida que no se considera la evaluación institucional por encima de la evaluación docente o la autoevaluación de las carreras, no se considera la evaluación de procesos o no existe la participación de los distintos actores en el diseño de la evaluación.

Por otro lado, la participación docente en la reforma es limitada. En las unidades académicas la reforma aparece, pese a los límites, como una oportunidad que no ha podido ser aprovechada. Los debates son limitados y la visión estratégica no toma la fuerza necesaria, los problemas de tiempo para debatir, la escasa experiencia en procesos de reforma curricular, las dificultades de apropiación de la misma, atrapada en la lógica de una comisión, son todos obstáculos para generar la mejor reforma posible. Adicional a ello hay que sumar la fragmentación docente donde las instancias 
de representación no logran aglutinar a la mayoría docente ni legitimarse ante los mismos. La ADUNAH por ejemplo pese a mantener posiciones críticas y de luchar por intereses de los docentes (principalmente legales y de opinión publica), no logra el reconocimiento del conglomerado docente. El miedo en un contexto de tendencias autoritarias, el individualismo docente, las diferencias ideológicas, así como las amenazas hacia la proletarización docente, conspiran para una organización y/o para mantener una posición coherente y unificada sobre la orientación de la reforma.

En este contexto las autoridades universitarias se organizaron jornadas informativas en el año 2014, con algunas facultades y centros, es decir, exclusivamente con docentes de las mismas facultades y centros. El propósito era precisamente informar (más que debatir) de lo que se había venido haciendo y lo que está por hacerse en la universidad según la perspectiva de las autoridades.

Las jornadas son positivas porque en la primera gestión de la actual rectora, en parte por la crisis y por el estilo de conducción, no se había logrado ningún tipo de acercamiento con los docentes. Por otro lado no se sabe hasta donde, como ha ocurrido en otros momentos y situaciones, son las críticas a la actual gestión las que obligaron y forzaron estas jornadas.

Uno de los puntos que presentaron las autoridades es sobre las condiciones y de donde debería surgir la reforma. En relación a la idea de que las propuestas deben surgir de las unidades académicas es en parte cierto y en buena medida quizá no ha sido bien aprovechada; quizá esta situación este asociada con la limitada claridad del proyecto de reforma en tanto visión colectiva, y porque la inercia de lo cotidiano se impone. Sin embargo, lo anterior no deja de tener su contradicción porque se dice que los docentes tienen que hacer la reforma pero ya existe una propuesta de autoevaluación y de reforma curricular que hay que seguir. Que alcance puede tener en estas condiciones la reforma y en qué medida el proceso será convergente con un proyecto de universidad. Se dispondrán los recursos y se tomaran las decisiones correspondientes a las propuestas de las unidades académicas.

\section{HACIA UNA UNIVERSIDAD DE ALTERNATIVAS.}

En el debate internacional un grupo de especialistas sobre el tema proponen algunos desafíos en los que hay acuerdos en América Latina y el Caribe. Estas perspectivas coinciden con varias ideas de los actores universitarios y con la perspectiva que se asume en este trabajo donde el tema político y la función social de la universidad son centrales. El pluralismo ideológico desde la interacción con actores sociales es una perspectiva valida dadas las tensiones internas de la UNAH y las exigencias de una realidad política del país que difícilmente acepta posiciones 
unilaterales. El pluralismo es convergente con la necesidad de acuerdos y pactos que el proceso de cambio de la universidad requiere. Sin embargo lo anterior no niega la posibilidad estratégica y urgente que supone las exigencias de una opción preferencial por lo pobres.

Uno de temas que Mollis plantea, como se puede observar, es el de la negociación y el consenso coincidente con la idea de pactos y acuerdos que en este trabajo se ha propuesto. Es necesario reconocerse para cambiar. Este es un tema central en un doble sentido: porque permite ver el carácter de la crisis que hay que definir y por otro, plantear los temas sobre los cuales reconstruir el futuro de la universidad. Como Sousa Santos también ha sostenido, de que es necesario definir en que consiste la crisis de la universidad y definir democráticamente que universidad se quiere. También es parte fundamental de este reconocimiento comprender y aceptar un encuentro universitario como forma innovadora para pactar la agenda del cambio e ir más allá de la idea tradicional /de que los liderazgos académicos van a realizar tales cambios.

Malagon (2005) por su parte plantea la presencia del conflicto en nuestras universidades, inevitable por el carácter de nuestras sociedades pero que hay que reconocer y orientar en perspectiva democrática, en clave de cumplir una función social relevante y en dialogo con los procesos de globalización. Es coincidente también con el papel político que se debe tener en la sociedad y la urgencia de formar profesionales socialmente responsables sin negar toda posibilidad de perspectivas plurales.

Ahora bien, el gran reto es la convergencia de actores y sus propuestas en un proceso de cambio o en la llamada reforma universitaria a condición de ser integradora desde los puntos aquí esbozados, porque tal como lo muestran algunas experiencias en América Latina, las reformas se han visto dificultadas por los límites en las estrategias de conducción.

Cuando el contexto es tan adverso como el actual donde no solo se impone la lógica del capital, sino también la tradición política, los procesos de cambio, como alternativa a la lógica excluyente y desigual del capital y de la dominación política, frente la crisis de convivencia, crisis ambiental y crisis de legitimidad universitaria, se tornan más difíciles pero paradójicamente son más urgentes.

Sobre algunas contribuciones de la universidad. Uno de los aportes que la universidad ha realizado en el periodo de la reforma es haber instalado las bases de un proceso de transición que supone niveles de fortalecimiento institucional al modo tradicional. La docencia funciona con regularidad, se hace investigación con 
mayor disponibilidad de recursos, se hace de la misma forma más vinculación, hay un manejo del presupuesto con un nivel de transparencia en tanto las inversiones son visibles, hay preocupación por los temas de la calidad de la educación universitaria que se observa en las medidas académicas tomadas, cada vez más hay una diversidad de eventos académicos y en general la universidad adquiere reconocimiento de la sociedad. Sin embargo hay que señalar que la falta de concertación de esos cambios y la exclusión de distintos actores de las decisiones en el rumbo de la institución, presentan una reforma limitada.

\section{La apuesta por el cambio sustantivo y concertado.}

En este estudio hay una preocupación y una apuesta por el cambio de y desde la universidad donde la dirección del cambio es muy importante. El camino recorrido de la universidad en la experiencia de la reforma en el periodo analizado conlleva el logro de un proceso de transición y de reformas curriculares en varias unidades académicas, pero es aún insuficiente para las exigencias de nuestra realidad en general y para las exigencias del cambio educativo que esa realidad demanda. Son reformas fragmentadas sin superar la tradición profesionalizante, son reformas que se ven solo como un producto pero que no valora los procesos. De ahí la apuesta por una universidad democrática y emancipadora que genera profesionales con los saberes pertinentes pero que también son mejores ciudadanos y sujetos que contribuyen creativamente a vivir mejor y a una vida que vale la pena ser vivida. Se requiere pasar de un cambio desde arriba y en parte desde afuera por la influencia del modelo internacional, a un cambio sostenido sobre la base de la participación y el acuerdo de los actores universitarios.

El cambio con participación permite darle una dirección concertada al mismo y por tanto hacerlo más sostenible en la medida que el conjunto de actores universitarios se comprometen con él. De esta forma el cambio contribuye a una gobernabilidad democrática. El debate y el acuerdo deben de extenderse hacia otros actores estratégicos externos como una forma de darle al cambio contenidos sustantivos sin menoscabar la autonomía universitaria. Implica además que la universidad debe fortalecerse como actor estratégico de la sociedad para incidir en los cambios que esta última requiere.

El cambio con el acuerdo de los actores universitarios principalmente y que conlleva la participación de los mismos como proceso, implica reconocer que el cambio sostenible y de carácter sustancial sin la subjetividad y la confianza de los agentes universitarios es débil cuando no imposible. La reforma curricular, el aula, la planificación académica participativa, el encuentro de facultades (como la ocurrida en Ciencias Sociales si asume los desafíos planteados por los participantes) 
y el encuentro universitario si se da (si el dialogo universitario se potencia a ese nivel de inclusión y se debate sobre la reforma necesaria) los claustros y el Consejo Universitario renovados, son entre otros, espacios importantes para la participación. En este sentido se recupera uno de los eslabones perdidos de la investigación educativa cuyos paradigmas dominantes solo consideraron productos educativos pero dejaron fuera los procesos donde la participación de los actores educativos y no educativos son fundamentales en el logro de tales productos. El cambio exige ser pedagogizado y el dialogo es criterio de verdad.

El cambio desde arriba ha estado orientado por variables externas (globalización del conocimiento desde la lógica del mercado) combinado con variables internas donde estas últimas (en este caso) son más decisivas aunque sin aprovechar este potencial en una dirección democrático emancipadora. Lo anterior permite observar también que el cambio de las universidades no solo es posible explicarlo desde variables externas sino que depende también de otras variables donde el papel de los actores universitarios es fundamental. Así también el cambio de la universidad es posible porque es resultado de construcciones sociales.

El cambio de la universidad por otro lado es la condición de posibilidad, en la actual coyuntura, para incidir en procesos de cambio en la sociedad. Es la idea de que si la universidad no cambia primero difícilmente podrá cambiar o aportar al cambio de la sociedad, eso es fundamental. En este sentido no se puede negar la voluntad política de cambio, lo que se cuestiona es la dirección y alcance del mismo y la forma de lograrlo. Si el cambio es complejo la participación de los actores contribuye en ese proceso aunque sea difícil concertar acuerdos. La autonomía permite que los actores universitarios debatan democráticamente cual es la mejor contribución a la sociedad. El conflicto de 2016 y los acuerdos firmados en tanto conlleven un cambio sustantivo de la universidad desde la apuesta democrático emancipadora, plantea la idea de que el cambio es posible porque las instituciones son construcciones sociales.

La ciudadanía y la formación de sujetos frente a la cultura autoritaria que prevalece en la sociedad y porque junto a la mejor formación disciplinar se requiere la formación de ciudadanos y sujetos con responsabilidad social, más las exigencias de la sociedad compleja que demanda ciudadanos y sujetos autónomos y reflexivos. Esta exigencia plantea experiencias en los espacios de formación que sean procesos participativos, acuerdos y pactos como escuelas de ciudadanía para el cambio democrático emancipador de nuestra sociedad.

El cambio sostenible y profundo en la dirección democrática emancipadora por la complejidad del mismo requiere pedagogizar el cambio lo que implica participación, acuerdos y pactos de los distintos actores. Una institución educativa 
como la universidad no puede y no debe eludir esta responsabilidad que es parte de su ethos.

Por otro lado es insuficiente buscar solo la gobernabilidad sino es a condición de que sea democrática. Si no adquiere esta condición y como una forma de procesar el conflicto, se corre el riesgo de regresiones e involuciones. Tomar decisiones solo con apego a la ley y cuando la representación no se ha conformado adecuadamente, es insuficiente.

En un contexto donde la formación para la ciudadanía y de sujetos asi como la proyección social pertinente, se insiste por tanto que un eje o una metodología clave del cambio son los acuerdos o pactos universitarios en su dimensión interna como exigencia de su dimensión externa. En este sentido Jose de Sousa (2010) ha sostenido que la "innovación relevante para un grupo de actores emerge de procesos de interacción social que incluyen su participación, lo que implica generar conocimiento significativo en el contexto de su aplicación e implicaciones".

Por tanto el cambio se sustenta en buena medida en la inclusión y en la participación de los actores lo que implica que la subjetividad cuenta, por tanto el cambio se basa en una "lógica de la confianza". Este es un reto para la universidad si se pretende lograr no solo un cambio sostenible sino también cambios más profundos. Con todo esto se trata de pedagogizar los procesos de cambio. Implica convertir la universidad en espacio público de deliberación y participación y desde aquí contribuir a la formación de sujetos y de ciudadanía.

SI se admite que la formación del sujeto y de la ciudadanía es el principal desafío de la educación, la reforma y los procesos de cambio deben considerar, como declarativamente se acepta por todos los actores, orientarse como ser orientadores de tales procesos. La ciudadanía y la formación de sujetos, como lo ha planteado Mollis, es una dimensión fundamental de la calidad de la educación. La generación de conocimiento útil como las habilidades para el empleo son tan importantes como la formación para la ciudadanía y la construcción de sujetos.

El tema de la ciudadanía y la formación del sujeto es algo que hay que coordinar con los otros niveles educativos. La formación de redes de capital social, redes de convivencia y cultura de paz, redes de solidaridad como de nueva relación con la naturaleza es fundamental pensarlas y realizarlas en conjunto con todo el sistema educativo. 
Las dimensiones que este sujeto y esta ciudadanía demandan para sí y para los otros se asocian con la capacidad de autonomía; con capacidad de análisis y de informarse, con capacidad de deliberar, incidir y participar en los temas y espacios de interés público; capacidad de construir comunidad democrática; responsabilidad social por el otro y por lo otro. Ello supone que todos los sujetos tienen condiciones materiales básicas, apuestan por una sociedad justa, luchan para que esto sea posible. Universitariamente todo esto requiere crear las condiciones para que este sujeto se desarrolle y se articule a la proyección social pertinente para potenciar los otros sujetos para la mayor humanización de unos y otros.

Uno de los aspectos sobre los que la universidad debe de poner atención es sobre el trabajo de regulación, en tanto "rectora" de la educación superior debe dar cuenta sobre el tipo de responsabilidad social de las universidades privadas y sobre la investigación que las mismas realizan o que sobre todo deben de hacer en función del mayor bienestar público.

Hay que potenciar por tanto el discurso y las prácticas de lo público que las autoridades universitarias han promovido. En función de lo público se debe regular la transnacionalización de la universidad, la definición de agendas o la cooperación internacional que las afecta.

Por otro lado colocar la universidad en perspectiva de transformación social con contribuciones de equidad y democratización importante, así como con aportes para construir nuevas reglas de convivencia, es una gran tarea que requiere la transformación misma de la universidad en condiciones de mayor democratización interna, situación que se convierte en el tema crítico del cambio. De ahí la idea de pactos y acuerdos, de la idea de universidad como espacio público de participación y deliberación.

La proyección social de la universidad es un espacio privilegiado para la formación de sujetos y de ciudadanos en la medida que se asume la responsabilidad por el otro (los excluidos principalmente o las víctimas de la violencia) y por lo otro (relaciones con la naturaleza) sin dejar de lado el dialogo de saberes. Internamente esta posibilidad ocurre en tanto la universidad se convierte en espacio público de deliberación y participación.

Los conflictos más recientes (julio 2015) que han tenido lugar en la universidad permiten revalorar las protestas estudiantiles, esta vez con la convergencia de frentes estudiantiles, asociaciones y movimientos independientes, como espacios de politización que ofrecen elementos para la construcción de ciudadanía. Y como los espacios tradicionales de participación no funcionan como política constructiva y 
positiva, aparece el conflicto como política negativa forzando la activación de estos y otros espacios. De esta forma se puede establecer que la ciudadanía es una conquista y que el conflicto es un lugar de la construcción de la ciudadanía y la conformación de los sujetos.

Así también en la medida que los estudiantes han sido los principales protagonistas de las protestas que se pueden considerar más radicales y quizá en parte porque han sido uno de los actores más afectados con las medidas tomadas por las autoridades en el periodo de la reforma, no solo han forzado un mayor debate, han urgido al dialogo como no se había imaginado y han planteado la necesidad de activar o crear espacios de participación. Todo ello redefine, si concretan estas exigencias, la reforma y plantea nuevos espacios de ciudadanía para todos los actores universitarios y en general para toda la comunidad universitaria. Los estudiantes se distancian así de lo dado y abren nuevas posibilidades, construyendo como sujetos colectivos nuevas realidades, abriendo horizontes de sentido. Activan de esta forma no solo la contingencia propia de la historia (Cerutti, 2006; 505), sino también la dimensión utópica como nuevas posibilidades.

Hay por tanto una ruptura de parámetros porque como dice Zemelmann (1998: 20) eso es posible porque todo fenómeno es un fenómeno histórico. Se potencian realidades y se construyen potencialidades como exigencias de sentido asociadas con la utopía. En otras palabras, aunque no solo eso, se activa la política que ha sido negada con las tendencias autoritarias. Y al ocurrir en el propio campus la dimensión utópica requiere ser integrada en el quehacer científico de la universidad.

Por otro lado, la formación de liderazgos es un tema central de la construcción de ciudadanía. Como aportar en ello es un gran desafío porque hasta la fecha la universidad contribuyo más a la formación de liderazgos tradicionales. En esta dirección los espacios de deliberación y participación son fundamentales. En relación a ello las protestas recientes estarían demandando la activación de dichos espacios.

Por su parte los conflictos de 2016 profundizan la idea de ciudadanía arriba planteada, pero también mantienen el conocimiento abierto en tanto se han generado debates sobre la reforma universitaria y sobre el papel de la universidad. En este sentido uno de los debates abiertos es sobre la contradicción entre derechos de minorías y derechos de mayorías. Por tanto si se admite que fueron minorías las que se tomaron la universidad, estas afectaron el derecho a tener clases de unas supuestas mayorías (hay que ver además hasta donde se trató de un consentimiento tácito de estas mayorías o de sectores de ella o no). Este es un argumento muy usado por las autoridades y en general de las elites para deslegitimar movimientos sociales. Ahora bien, si esto es así hay que reconocer que las acciones de protesta tienen efectos y que son considerados 
como los efectos no deseados de la acción. El derecho a la protesta sin embargo, por el carácter de la protesta, es una apuesta por el derecho a tener derechos de un nivel sustantivo por sobre derechos restringidos como la asistencia a clases. Los primeros suponen un proceso de formación de sujetos y ciudadanía en tanto se democratiza la reforma y se admite la participación autentica de los estudiantes en los órganos de representación lo que consecuentemente para los segundos se cualifica el asistir a clases colocándolas en un nivel de calidad más los nuevos derechos de participación como el derecho a tener derechos. Lo anterior también se justifica desde la idea que los derechos de minorías se activan como consecuencia de la negación de derechos por parte de las autoridades universitarias. Y sobre todo cuando no se resolvieron los conflictos de 2015 y no se previó la agudización de estos y la aparición de otros tantos.

Los acuerdos firmados por los estudiantes y las autoridades, para poner fin quizá a uno de los conflictos más largos de la historia de la universidad, son un gran avance para la construcción de ciudadanía y de sujetos, aunque dependerá del cumplimiento del mismo y en la medida que se creen las mejores condiciones para ello. Si se atienden las demandas democráticas será importante la participación de otros actores internos lo que consecuentemente previene futuros conflictos. Pero la participación democrática de los distintos actores requiere discutir los contenidos del cambio y al mismo tiempo la responsabilidad de construir comunidad universitaria con la mejor calidad profesional.

El conflicto por otro lado cuestiona los procesos de reforma y los replantea en otra dirección. Pero el cambio sustantivo requiere abrir la participación a otros actores como los docentes y administrativos para asegurar una mayor sostenibilidad del mismo. Indica también que el "dialogo de saberes" no solo es un tema externo sino también interno, entre actores universitarios.

\section{Sobre la necesaria proyección social y la pertinencia de la universidad.}

La universidad sigue siendo profesionalizante, orientada por la docencia. Cuando esto ocurre, según Ellacuria (1980), la docencia se vuelve repetitiva, abstracta y sin responsabilidad ética y social. En este sentido propone una universidad orientada por la proyección social. Lo anterior supone que la universidad gana legitimidad social, crea condiciones externas para la formación de ciudadanía y sujetos y fundamentalmente contribuye a ofrecer alternativas a las formas de exclusión, a la violencia y a la destrucción irracional de la naturaleza.

Al orientar el quehacer universitario desde la proyección social o en su defecto desde el equilibrio de las funciones tradicionales de la universidad, esta última aumenta 
su potencial de cambio y más si esa orientación asume una perspectiva de equidad y relevancia social desde una "opción preferencial por los pobres" que contribuya a mejorar las bases de una mejor convivencia social.

La universidad no se puede escudar en una supuesta excelencia o solo puede apelar a ella si a la vez considera y pone lo mejor de sí para luchar contra las desigualdades y las distintas formas de exclusión. La calidad está asociada no solo a la mejor formación profesional, sino también con la construcción de sujetos y de la ciudadanía así como con las contribuciones relevantes que ofrezca la universidad en la lucha contra las desigualdades. Desde aquí es fundamental la contribución de la universidad a la democratización del conocimiento.

Por otro lado, si todo lo anterior tiene un énfasis en la pertinencia social, esta debe de ser complementada con una colaboración estrecha con el Estado y su fortalecimiento así como contribuciones estratégicas con el sector productivo.

\section{BIBLIOGRAFÍA}

Brunner, Joaquin (2007). Universidad y sociedad en américa Latina. Universidad veracruzana, Biblioteca Digital de Investigación Educativa.

Cerutti, Horacio (2006). Filosofía de la Liberación Latinoamericana. FCE, México.

De Sousa Santos Boaventura (2005). La universidad del siglo XXI. Para una reforma emancipatoria y democrática. UNAM.

De Souza José. (2010). La investigación científica para el desarrollo y el desarrollo de la investigación científica. Geopolítica del conocimiento y los aportes de la ciencia a la gestión del "desarrollo" en América Latina. UNAH, Doctorado en Gestión del Desarrollo.

Dias Sobrinho (2008). “cambios y reformas en educación superior”. En :Tunnermann, Carlos (edit). La educación superior en américa latina, UNESCO, Universidad Javeriana, IESALC, Colombia.

Dussell. Enrique (2011). Filosofía de la Liberación, FCE, México

Ellacuria, Ignacio (1980). "Universidad y política". ECA, núm. 383, Universidad Centroamericana, El Salvador.

Mc laren, Peter y Huerta Luis (2010). "El cambio educativo, el capitalismo global 
y la pedagogía critica revolucionaria” Revista Mexicana de Investigación educativa núm. 47 , oct-dic.

Malagon, Luis (2005). "Cambios y conflictos en los discursos político pedagógicos sobre la universidad”. Archivos Analíticos de Políticas Educativas, Vol 13, Arizona State University.

Molina, María Mercedes (2008). Introducción al estudio de la universidad en Latinoamérica,latinoam.estud.educ. Manizales (Colombia), 4 (1): 129 - 142, enero - junio.

Las mascaras del saber en las universidades latinoamericanas"www.bdigital.unal. edu.co/7386/1/marcelamollis.20121.pdf (fecha de consulta, julio 23 de 2014).

Posas, Mario (2014). Los hitos históricos de la universidad. Editorial Universitaria, Tegucigalpa.

Quintero y otros (2010). “El cambio conducido en la universidad: la percepción de los académicos”. Revista de Educación Superior núm. 155, Julio-septiembre, México.

Tunermann, Carlos (2001). Universidad y sociedad. Balance histórico y perspectivas desde América Latina. Edit, Hispamer, Managua.

Zemelman, Hugo (2011). "Implicaciones epistémicas del pensar histórico desde la perspectiva del sujeto”. Desacatos, núm. 37, IPECAL, México. 